\title{
Non-Positivity of the Wigner Function and Bounds on Associated Integrals
}

\author{
A.J. Bracken ${ }^{1, a}$, D. Ellinas ${ }^{2, b}$ and J.G. Wood ${ }^{1, c}$ \\ ${ }^{1}$ Centre for Mathematical Physics, Department of Mathematics \\ University of Queensland, Brisbane 4072, Australia \\ 2 Department of Sciences, Section of Mathematics \\ Technical University of Crete, GR-731 00 Chania, Crete, Greece \\ Paper presented at the Wigner Centennial Conference, \\ Pécs, Hungary, July, 2002 \\ (to appear in the Proceedings)
}

\begin{abstract}
The Wigner function shares several properties with classical distribution functions on phase space, but is not positive-definite. The integral of the Wigner function over a given region of phase space can therefore lie outside the interval $[0,1]$. The problem of finding bestpossible upper and lower bounds for a given region is the problem of finding the greatest and least eigenvalues of an associated Hermitian operator. Exactly solvable examples are described, and possible extensions are indicated.
\end{abstract}

\section{Introduction}

Since its introduction [1] in 1932, the Wigner distribution function has proved an important tool in many areas of quantum physics and chemistry. Recently, in quantum tomography [2, the Wigner function has been reconstructed from experimentally measured probabilities associated with a quantum system in a definite state. Given a finite amount of data, it is clear that such a reconstruction must inevitably be limited to a finite region of phase space. 
And here there arises a problem peculiar to quantum tomography. In the classical case, if the probability density has been reconstructed on a finite region, and if its integral over that region, the 'probability mass' on the region, is close to 1 , then it is certain that the density on the remainder of phase space is small, in the sense that the probability mass there must be small. But in the quantum case, because the Wigner function takes negative as well as positive values in general, the 'quasiprobability mass' on regions of phase space outside one particular region may be large positive or negative, even when the quasiprobability mass on that particular region is known to be close to 1 . This is the primary motivation for our study, which aims to provide best possible upper and lower bounds on the integral of the Wigner function over any given region $S$ of phase space.

In previous work [3], it has been shown that this is equivalent to finding the greatest and least eigenvalues of a corresponding Hermitian operator $\widehat{S}$, which we refer to as the 'region operator' for $S$. It is an operator analogue of the classical characteristic function $\delta_{S}$, which has the value 1 for points inside $S$, and 0 for points outside. Here we describe some exact results and the basis on which they can be obtained, and indicate some directions of possible generalization.

\section{Mathematical background and results}

For a system with one degree of freedom, the invertible mapping from functions $A(q, p)$ on the phase plane $\Gamma$ to linear operators $\widehat{A}$ on Hilbert space is accomplished with the help of Weyl's kernel operator [4,

$$
\begin{aligned}
\widehat{W}(q, p) & =\frac{1}{(2 \pi \hbar)^{2}} \int_{\Gamma} e^{i\left[p^{\prime}(q-\hat{q})-q^{\prime}(p-\hat{p})\right] / \hbar} d q^{\prime} d p^{\prime} \\
\widehat{A}=\int_{\Gamma} A(q, p) \widehat{W}(q, p) d q d p, & A(q, p)=2 \pi \hbar \operatorname{Tr}(\widehat{\mathrm{A}} \widehat{W}(\mathrm{q}, \mathrm{p}))
\end{aligned}
$$

Here $\operatorname{Tr}$ denotes the trace, and $\hat{q}, \hat{p}$ are canonical operators. The generalizations to many degrees of freedom are obvious. From this point we work with dimensionless variables, in effect setting $\hbar=1$.

As special cases, we have the Wigner function $W_{\rho}(q, p)$ coresponding to the operator $\widehat{\rho} /(2 \pi)$, where $\widehat{\rho}$ is the density operator defining the state of the

quantum system; and the region operator $\widehat{S}$, corresponding to the characteristic function $\delta_{S}(q, p)$ of a given 2-dimensional region $S \in \Gamma$ :

$W_{\rho}(q, p)=\operatorname{Tr}(\widehat{\mathrm{W}}(\mathrm{q}, \mathrm{p}) \widehat{\rho}), \quad \widehat{\mathrm{S}}=\int_{\Gamma} \delta_{\mathrm{S}}(\mathrm{q}, \mathrm{p}) \widehat{\mathrm{W}}(\mathrm{q}, \mathrm{p}) \mathrm{dq} \mathrm{dp}=\int_{\mathrm{S}} \widehat{\mathrm{W}}(\mathrm{q}, \mathrm{p}) \mathrm{dq} \mathrm{dp}$. 
In the last step we have used the defining property of the characteristic function.

Given a smooth contour $C \in \Gamma$, it is natural to define also an associated 'contour operator' $\widehat{C}$ by

$$
\widehat{C}=\int_{\Gamma} \widehat{W}(q, p) \delta_{C}(q, p) d q d p
$$

Here $\delta_{C}(q, p)$ is the 'characteristic (generalised) function' for the contour, defined by the property that, for every smooth function $F$ on $\Gamma$,

$$
\int_{\Gamma} F(q, p) \delta_{C}(q, p) d q d p=\int_{C} F d l
$$

where $d l$ the element of length on $C$. Then we can write

$$
\widehat{C}=\int_{C} \widehat{W} d l
$$

For example, if the contour is defined by the graph of a simple function, such as $p=F(q), a<q<b$, then $\delta_{C}(q, p)=\delta(p-F(q))$ for $a<q<b$, and $\delta_{C}(q, p)=0$ elsewhere. Going one dimension further down, we can define the 'point operator'

$$
\widehat{P}=\int_{\Gamma} \delta_{P}(q, p) \widehat{W}(q, p) d q d p=\widehat{W}\left(q_{0}, p_{0}\right)
$$

corresponding to the point $\left(q_{0}, p_{0}\right) \in \Gamma$. The characteristic function for the point, $\delta_{P}(q, p)=\delta\left(q-q_{0}\right) \delta\left(p-p_{0}\right)$, is more singular than that for a contour $C$, which is more singular than that for a 2-dimensional region $S$ in the plane. Clearly these notions can be generalized to regions $R$ of dimensions $2 f, 2 f-1, \ldots, 0$ in the $2 f$-dimensional phase space of a system with $f$ degrees of freedom, with the introduction of corresponding characteristic functions $\delta_{R}$ of increasing singularity, and corresponding region operators $\widehat{R}$.

Critical to what follows is Wigner's observation [1] that, when the quantum system is in the state $\widehat{\rho}$, the mean value of any operator (such as $\widehat{R}$ ) is given in terms of the corresponding phase space function (in this case $\delta_{R}$ ) and the Wigner function, by

$$
\langle\widehat{R}\rangle=\int_{\Gamma} \delta_{R} W_{\rho} d \Gamma=\int_{R} W_{\rho} d V,
$$

where $d V$ is the volume element in $R$. Since the expectation value $\langle\widehat{R}\rangle$ must lie between the greatest and least eigenvalues of $\widehat{R}$ (or more generally, between 
the best possible upper and lower bounds on its spectrum), the same must be true of the last integral in (17). Moreover, the bounds are attained (or more generally, approached arbitrarily closely) if $\widehat{\rho}$ is a pure state corresponding to the associated (generalized) eigenvector of $\widehat{R}$. In short, if we can solve the eigenvalue problem for $\widehat{R}$, we have best possible bounds on the last integral in (17), and we know when and how those bounds are attained.

The circular disk of radius $a$, centred on the origin in the phase plane is invariant under canonical transformations generated by the classical harmonic oscillator Hamiltonian $H=p^{2}+q^{2}$, and it follows that the corresponding region operator $\widehat{S}_{a}$ commutes with the quantum oscillator Hamiltonian operator, and has the same eigenvectors, namely the number states $|n\rangle, n=0,1, \ldots$. This is significant for quantum tomography, where such states are amongst the easiest to measure [2]. The eigenvalue of $\widehat{S}_{a}$ on $|n\rangle$ is [3]

$$
\lambda_{n}(a)=(-1)^{n} \int_{0}^{a^{2}} L_{n}(2 u) e^{-u} d u
$$

where $L_{n}$ is the Laguerre polynomial. For a given $a$, it is then possible to see which eigenvalue is smallest (say $\lambda_{N}(a)$ ), and which is largest (always $\lambda_{0}(a)$ ), and hence what are the lower and upper bounds on the integral of the Wigner function over the disk. These bounds are attained when the system is in the pure state $|N\rangle$ or $|0\rangle$, respectively. The eigenvalues of the contour operator $\widehat{C}_{a}$ corresponding to the boundary of the circular disk are, again on the number states, $\mu_{n}(a)=d \lambda_{n}(a) / d a$. There is a remarkable Lie-algebraic structure [5] associated with these spectra.

For the contour operator $\widehat{C}_{L}$ corresponding to a straight line segment of length $L$ in the phase plane, say the segment along the $p$-axis from $p=-L / 2$ to $p=L / 2$, we find [6] a continuous spectrum and generalized eigenfunctions:

$$
\mu(k)= \pm \frac{1}{k \pi} \sin (k L),(0 \leq k<\infty) ; \quad \varphi_{k}(x)=\frac{1}{\sqrt{\pi}}\left\{\begin{array}{c}
\cos (k x) \\
\sin (k x)
\end{array} .\right.
$$

This segment is invariant under canonical transformations generated by the free-particle Hamiltonian $H=p^{2}$, and also under the parity transformation which sends $q, p$ to $-q,-p$, hence the appearance of the common eigenfunctions of the quantum free-particle Hamiltonian operator and parity operator in (9).

From these simple results for disks, circles and straight segments we can build up results for more complicated regions in phase spaces of higher dimensions. For example, in the 4-dimensional phase space of a 2-dimensional quantum system, we can find best possible upper and lower bounds on the integral of the Wigner function on the rectangle $-L_{1} / 2<q_{1}<L_{1} / 2$, 
$-L_{2}<q_{2}<L_{2} / 2$ within the 'Poincaré section' $p_{1}=p_{2}=0$; or on the cylinder $q_{1}^{2}+p_{1}^{2} \leq a^{2},-L / 2<p_{2}<L / 2, q_{2}=0$; or on its surface, and so on.

Investigations continue towards a "spectral theory" of quasiprobability mass for bosonic systems, and into analogous results for spin and other finite dimensional quantum systems, with expected applications in quantum tomography.

\section{Notes}

Email:

a. ajb@maths.uq.edu.au b. ellinas@science.tuc.gr c. jgw@maths.uq.edu.au

\section{References}

[1] E.P. Wigner, Phys. Rev. 40 (1932), 749.

[2] U. Leonhardt, Measuring the Quantum State of Light (Cambridge University Press, Cambridge UK, 1997).

[3] A.J. Bracken, H.-D. Doebner and J.G. Wood, Phys. Rev. Lett. 83 (1999), 3758.

[4] H. Weyl, Z. Phys. 46 (1927), 1.

[5] A.J. Bracken, D. Ellinas and J.G. Wood, Group Theory and Quasiprobability Integrals of Wigner Functions (quant-ph/0304010), to appear in J. Phys. A.

[6] A.J. Bracken, D. Ellinas and J.G. Wood (in preparation). 